\title{
Radiobiological mechanisms of stereotactic body radiation therapy and stereotactic radiation surgery
}

\author{
Mi-Sook Kim, MD, PhD', Wonwoo Kim, PhD¹, In Hwan Park, BA', Hee Jong Kim, MS', Eunjin Lee, MS', \\ Jae-Hoon Jung, PhD', Lawrence Chinsoo Cho, MD², Chang W. Song, PhD² \\ 'Research Center for Radiotherapy, Korea Institute of Radiological and Medical Sciences, Seoul, Korea; \\ ${ }^{2}$ Department of Radiation Oncology, University of Minnesota Medical School, Minneapolis, MN, USA
}

\begin{abstract}
Despite the increasing use of stereotactic body radiation therapy (SBRT) and stereotactic radiation surgery (SRS) in recent years, the biological base of these high-dose hypo-fractionated radiotherapy modalities has been elusive. Given that most human tumors contain radioresistant hypoxic tumor cells, the radiobiological principles for the conventional multiple-fractionated radiotherapy cannot account for the high efficacy of SBRT and SRS. Recent emerging evidence strongly indicates that SBRT and SRS not only directly kill tumor cells, but also destroy the tumor vascular beds, thereby deteriorating intratumor microenvironment leading to indirect tumor cell death. Furthermore, indications are that the massive release of tumor antigens from the tumor cells directly and indirectly killed by SBRT and SRS stimulate anti-tumor immunity, thereby suppressing recurrence and metastatic tumor growth. The reoxygenation, repair, repopulation, and redistribution, which are important components in the response of tumors to conventional fractionated radiotherapy, play relatively little role in SBRT and SRS. The linear-quadratic model, which accounts for only direct cell death has been suggested to overestimate the cell death by high dose per fraction irradiation. However, the model may in some clinical cases incidentally do not overestimate total cell death because high-dose irradiation causes additional cell death through indirect mechanisms. For the improvement of the efficacy of SBRT and SRS, further investigation is warranted to gain detailed insights into the mechanisms underlying the SBRT and SRS.
\end{abstract}

Keywords: Stereotactic body radiation therapy, Stereotactic radiation surgery, Radiobiology

\section{Introduction}

An increasing number of cancer patients are treated with stereotactic body radiation therapy (SBRT) and stereotactic radiation surgery (SRS) in recent years [1-6]. The significant paradigm shift from the conventional multi-fractionated radiotherapy to hypo-fractionated SBRT and SRS has been possible owing to the remarkable technological advances for tumor imaging and also for radiation delivery systems. Contrarily to such technological progress, the biological mechanisms of SBRT and SRS have been elusive [7-11]. In this review, we have described experimental evidence that indirect tumor cell death due to vascular damage and ensuing immune responses may play crucial roles in the response of tumors to SBRT and SRS [7,8,11-15].

Received 26 November 2015, Revised 7 December 2015, Accepted 7 December 2015.

Correspondence: Chang W. Song, PhD, Department of Radiation Oncology, University of Minnesota Medical School, 420 Delaware St. SE, Mayo Mail Code 494, MN 55455, USA. Tel: +1-612-626-6852, Fax: +1-612-624-5445, E-mail: Songx001@ umn.edu

(c) This is an Open Access article distributed under the terms of the Creative Commons Attribution Non-Commercial License (http://creativecommons.org/ licenses/by-nc/4.0/) which permits unrestricted non-commercial use, distribution, and reproduction in any medium, provided the original work is properly cited.

www.e-roj.org 


\section{Direct Tumor Cell Death Cannot Account for the Efficacy of SBRT and SRS}

In treating intracranial or extracranial disorders with SRS, the targets are irradiated with 15-25 Gy of radiation in 1-2 fractions, and treating extracranial tumors with SBRT, tumors are treated with 30-60 Gy of irradiation in 2-5 fractions. It has been known that there are as many as $10^{8}-10^{9}$ cells in a $1 \mathrm{~g}$ tumor implying that 8-9 log tumor cells should be

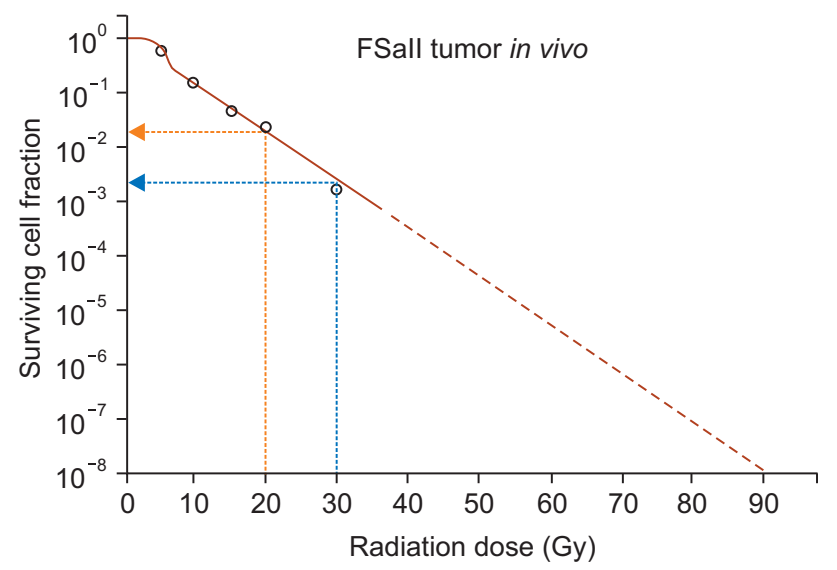

Fig. 1. Radiation survival curve of FSall tumor cells in vivo. Tumors grown s.c. in the legs of $\mathrm{C} 3 \mathrm{H}$ mice were irradiation with 5 to $30 \mathrm{~Gy}$ in a single dose and the number of clonogenic cells in each tumor was determined with in vivo-in vitro excision assay method. The surviving cell fractions in the irradiated tumors was obtained by normalizing the number of clonogenic cells in irradiated tumors against that in unirradiated control tumors. It is shown that $20 \mathrm{~Gy}$ and $30 \mathrm{~Gy}$ irradiation in a single dose decreased the cell survival to about $10^{-2}$ and $10^{-3}$, respectively. The extension of the survival curve shows that $90 \mathrm{~Gy}$ irradiation is needed to reduce the cell survival to $10^{-8}$. eradicated to control $1 \mathrm{~g}$ tumors [16]. A relevant question is whether the radiation doses used in SRS and SBRT are sufficient to eradicate all the tumor cells in the targets. Fig. 1 shows the radiation survival curve of FSall fibrosarcoma grown subcutaneously in the legs of $\mathrm{C} 3 \mathrm{H}$ mice [17]. Like many other experimental tumors and human tumors, about 20\% of the clonogenic cells in FSall tumors are hypoxic cells. The initial rapid decrease in the cell survival corresponds to the death of radiosensitive oxic cells, and the subsequent gradual decrease in cell survival reflects the death of radioresistant hypoxic cells. It is demonstrated that the irradiation with 20-30 Gy in a single dose could kill tumor cells only by 3-4 logs and that irradiation with doses as high as $90 \mathrm{~Gy}$ is needed to reduce cell survival by 8 logs. Leith et al. [18] concluded that, if the radiation-induced tumor cells are assumed due only to DNA double-strands break, a single dose irradiation with doses as large as 80-90 Gy is needed to control $3 \mathrm{~cm}$ diameter brain tumors assuming 20\% of the tumor cells are hypoxic cells. This estimated radiation dose to control brain disorders are far greater than 15-25 Gy applied in the highly effective SRS [6]. The calculation by Brown et al. [19] using conventional radiobiological principles also indicated that the radiation doses used in SBRT are significantly insufficient to kill all the clonogenic cells in 1-3 cm diameter tumors, assuming 10\%-20\% of tumor cells are hypoxic. However, clinical SBRT with 30-60 Gy in 2-5 fractions are highly effective to control various tumors as larger as 5-6 cm diameters [2] (Fig. 2). It is therefore evident that the conventional radiobiological cell killing mechanisms that radiation directly kills tumor cells by causing DNA double-strand breaks alone cannot account for the highly effective SRS and SBRT $[7,8,10-15]$. In this respect, recent emerging evidence strongly indicates that SBRT and SRS are effective because, in addition to direct cell death, indirect
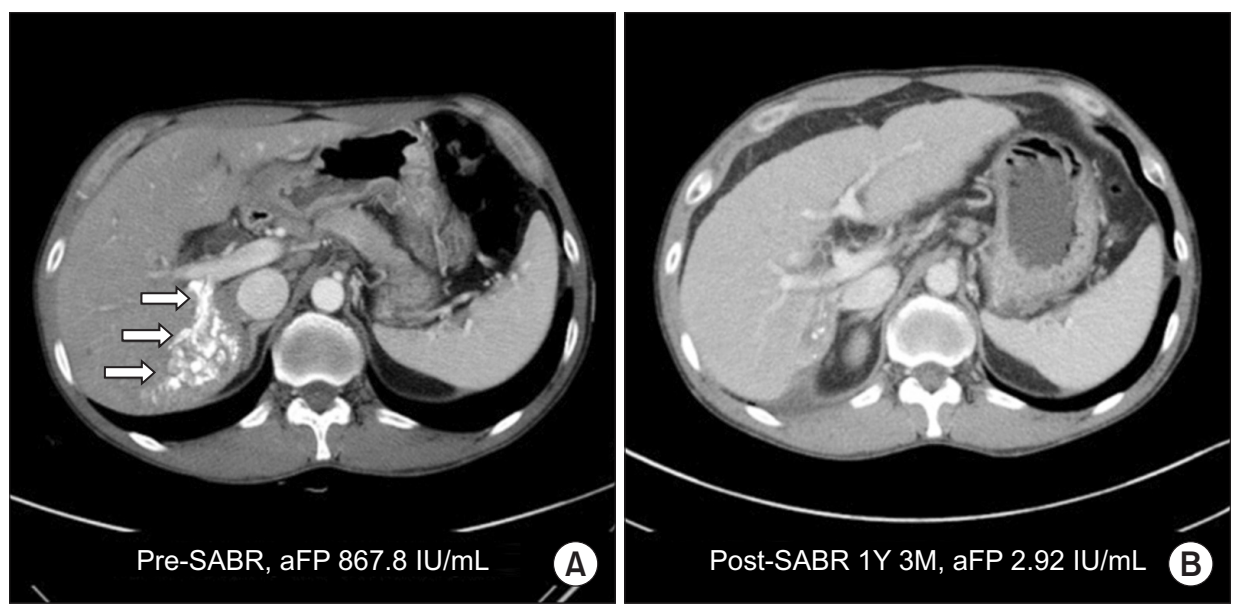

Fig. 2. The response of a human hepatocarcinoma to stereotactic body radiation therapy with $48 \mathrm{~Gy}$ in 3 fractions of 16 Gy applied with 3 days intervals. $(A, B)$ The computed tomography scan taken 1 year and 3 months after the treatment indicates the tumor was almost completely eradicated (arrow). SABR, stereotactic ablative radiotherapy; aFP, alphafetoprotein. 
cell death through vascular damage occurs when tumors are exposed to high dose hypo-fractionated irradiation [10-15].

\section{High Dose Hypo-fractionated Irradiation Causes Vascular Damage in Tumors}

The increasing needs for vital nutrients including oxygen in growing tumors stimulate formation of new tumor blood
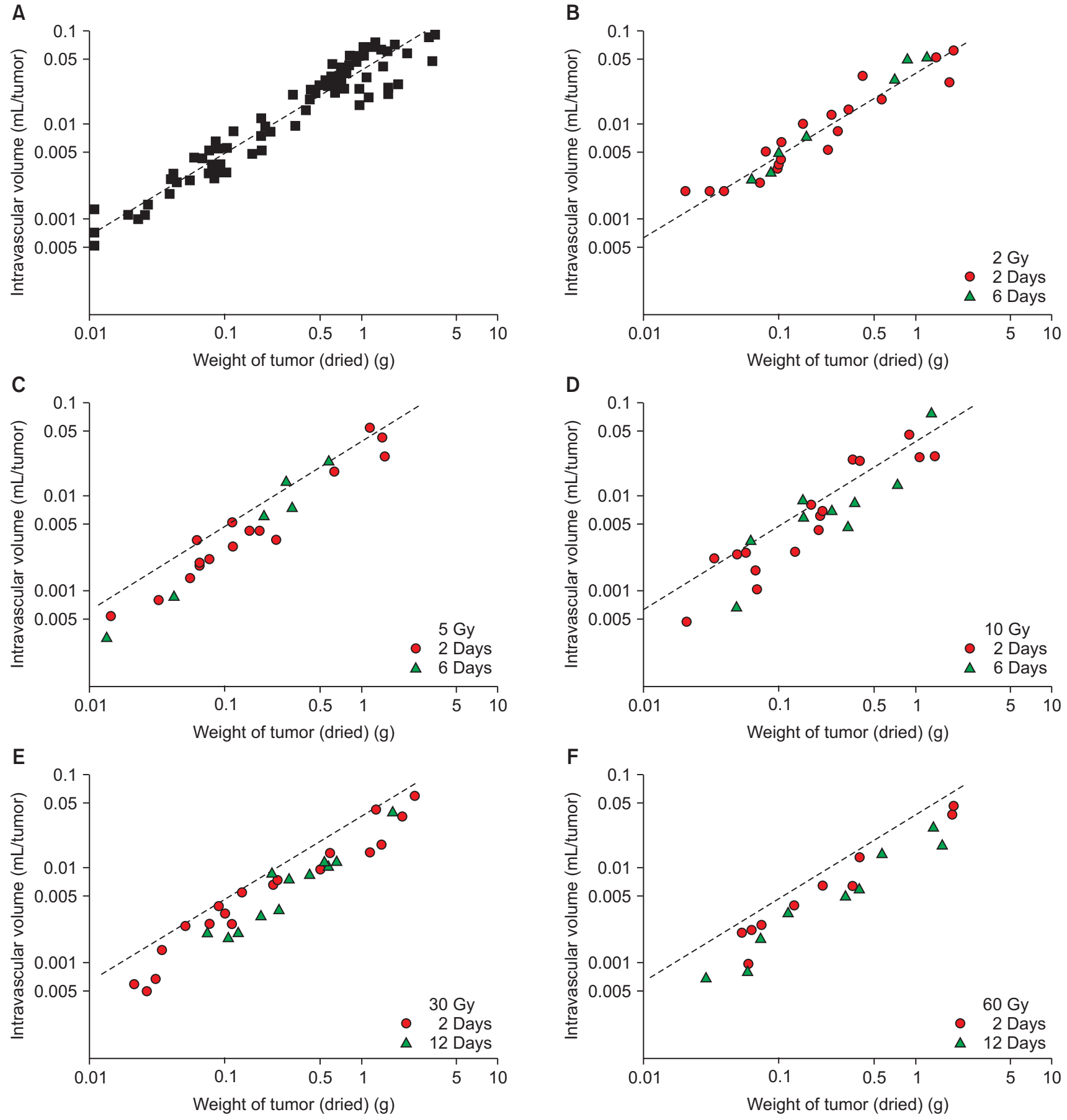
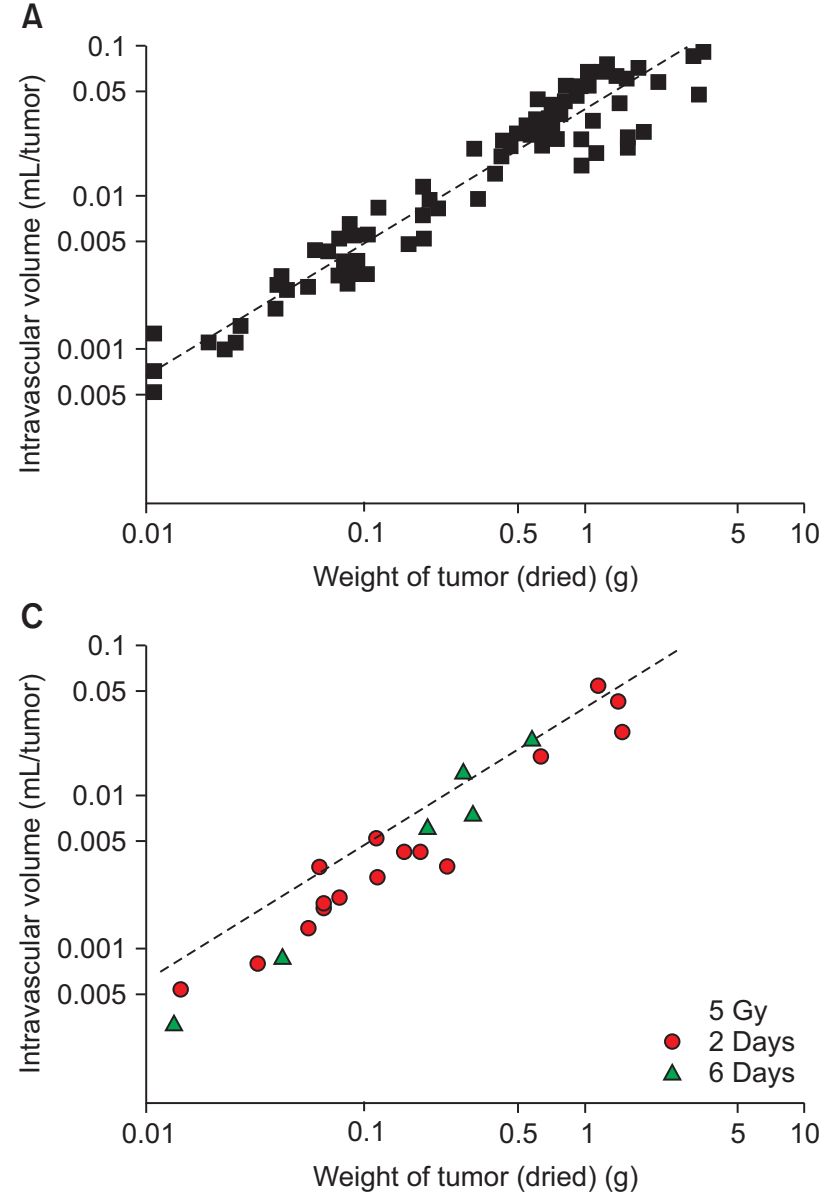
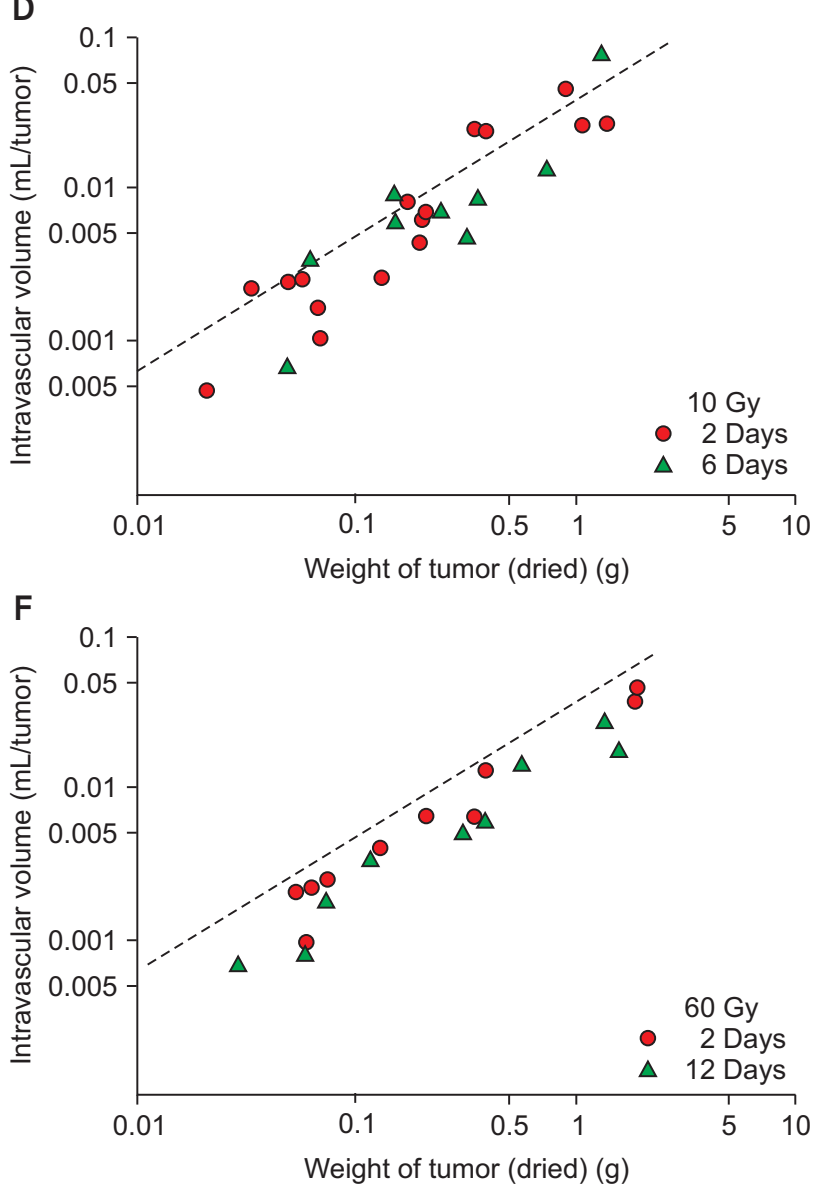

Fig. 3. (A-F) The functional intravascular volume in 0.1-5.0 gram (g) Walker tumors in the flank of rats after 2-60 Gy irradiation in a single exposure. The dotted lines are the vascular volume in the unirradiated control tumors of various sizes as shown in Fig. 2A. Dose dependent decline in functional vascular volume occurred at 2-12 days after irradiation. 
vessels [20]. The newly and hastily formed capillary-like tumor blood vessels are comprised of a single layer of endothelial cells supported by incomplete and disorganized basement membranes and pericytes. These newly formed tumor blood vessels are not only structurally but also functionally abnormal [20]. The irregularly shaped tumor endothelial cells are loosely connected to others with wide gaps between them, which are frequently plugged by tumor cells. The abnormal tumor blood vessels are rather leaky probably due in part to the gaps between endothelial cells and also due to the incomplete support of basement membrane [21]. Although little is known about the effects of high dose hypo-fractionated irradiation such as SBRT or SRS on human tumor vasculatures, a wealth of information has been accumulated on the effects of high dose hypo-fractionated irradiation on the vasculatures of experimental tumors. This subject has been discussed in detail in a recent review article [15]. In the 1970s, Song and his associates $[17,22-26]$ conducted a series of experiments to reveal the effects of $X$-irradiation on the vascular functions in experimental rodent tumors using radioisotope methods. In Walker tumors grown s.c. in the legs of rats, X-irradiation with 2.5-10 Gy caused a rapid and progressive decline in the functional vascularity, i.e., circulating blood volume, within several hours [25]. After hitting nadir in 5-6 hours, the functional vascularity recovered in 18-24 hours in the tumors irradiated with 2.5 or $5.0 \mathrm{~Gy}$, but not in the tumors irradiated with $10 \mathrm{~Gy}$. As shown in Fig. 3, irradiation of Walker tumors with 5 or 10 Gy caused measurable decreases in the functional intravascular volumes in 2 and 6 days [24]. Likewise, the functional vascular volumes of Walker tumors 2 and 12 days after irradiation with 30 or $60 \mathrm{~Gy}$ in a single exposure were markedly less than that of control tumors. The functional vascular volume in Walker tumors irradiated with 30 Gy began to recover from 15-16 days post-irradiation, concomitantly with the regrowth of tumors. Importantly, the extravasation of plasma protein, i.e., vascular permeability, significantly increased in 2 hours after irradiation while the vascular volume decreased. Emami et al. [27] also found that the blood flow in rhabdomyosarcoma grown in the scalp of rats declined by $40 \%-50 \%$ within 2 hours after irradiation with 16.5-60.5 Gy in a single dose, but the blood flow in the tumors irradiated with 16.5 Gy recovered by 24 hours. Brown et al. [28] recently reported that irradiation of human glioma xenografts in rat brain with $15 \mathrm{~Gy}$ caused a rapid decrease in blood flow as measured with magnetic resonance imaging followed by recovery in several hours. This study, unfortunately, did not investigate whether the recovery in blood flow was temporal or permanent. Nevertheless, the rapid decline in blood flow or vascularity after high dose irradiation observed in Walker tumors of rats [25], rhabdomyosarcoma of rats [27] and human glioma xenograft [28] is of interest. One conceivable cause of such early declines in tumor blood flow after irradiation is the rapid increase in extravasation of plasma protein due to an increase in vascular permeability $[24,26]$, which would increase the intratumor fluid pressure and compress the capillary-like tumor blood vessels. GarciaBarros et al. [29] reported that irradiation of tumors with doses higher than 8-10 Gy rapidly caused ceramide-mediated apoptotic death in endothelial cells, thereby leading to vascular occlusion and tumor cell death. Park et al. [15] reported that the endothelial cells harvested from human breast cancer were significantly more radiosensitive than the endothelial cells from the adjacent normal breast tissues. Solesvik et al. [30] reported that irradiation of human melanoma xenografts with 10-15 Gy impaired 35\%-45\% of 5-15 $\mu \mathrm{m}$ diameter blood vessels and $20 \mathrm{~Gy}$ irradiation destroyed $50 \%$ of 25 to $35 \mu \mathrm{m}$ diameter vessels in a week (Fig. 4A). Using three-dimensional high-frequency power Doppler ultrasound image, El Kaffas et al. [31] recently observed that 16 Gy irradiation reduced the blood perfusion in human breast cancer xenografts to less than $50 \%$ of control in 24 hours (Fig. 4B). Irradiation of human glioblastoma xenografts grown in the brain of nude mice with 15 Gy reduced the tumor blood perfusion to $10 \%$ of control in 2 weeks [32]. In mouse prostate tumors grown in the thigh of mice, irradiation with $25 \mathrm{~Gy}$ in a single dose decreased the tumor microvascular density over 3 weeks to nadirs of 25\% [33]. In a recent study with FSall tumors grown s.c. in $\mathrm{C} 3 \mathrm{H}$ mice, irradiation with $20 \mathrm{~Gy}$ cause a marked vascular occlusion and increase in the expression of hypoxia-inducible factor 1- $\alpha$ (HIF-1 $\alpha$ ) and vascular endothelial growth factor (VEGF), which could be attributed to an increase in hypoxia [13]. Fig. 5 also shows that irradiation of FSall tumors with 20 Gy markedly reduced blood perfusion in 2 days as determined with Hoechst 33342 perfusion method. It is relevant to point out that the blood vessels in xenografts are host origin, mouse, and thus the radiation-induced vascular changes in xenografts may not depict the possible vascular changes in human tumors. Nevertheless, these numerous reports on the radiationinduced vascular changes in tumors may be summarized as follows: the vascular damages in tumors by 5-10 Gy in a single exposure is relatively moderate whereas irradiation with doses higher than $10 \mathrm{~Gy}$ per fraction induces severe and long-lasting vascular occlusions. It is important to note that in contrast to the tumor blood vessels, the blood vessels in normal tissue 


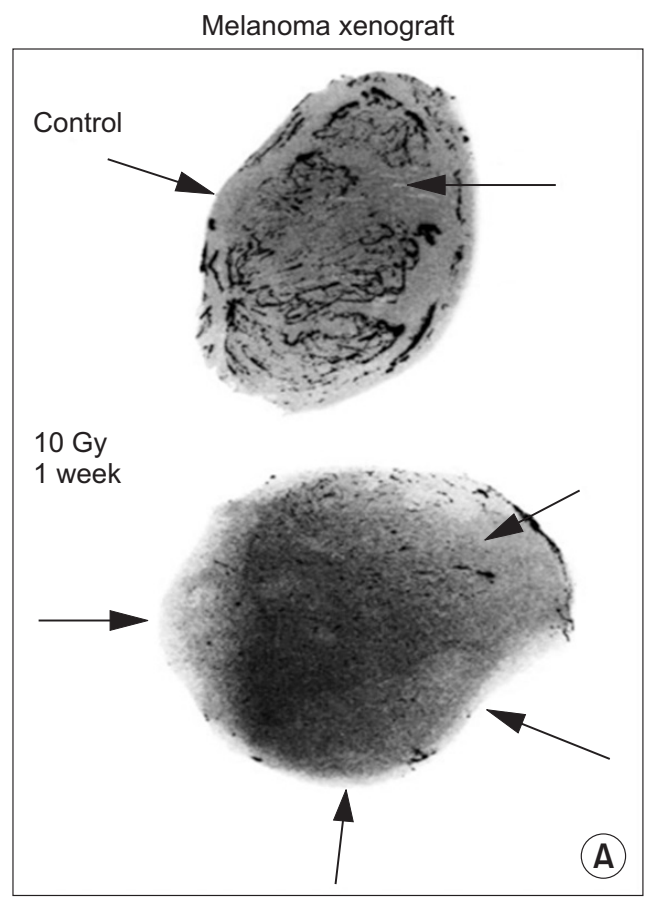

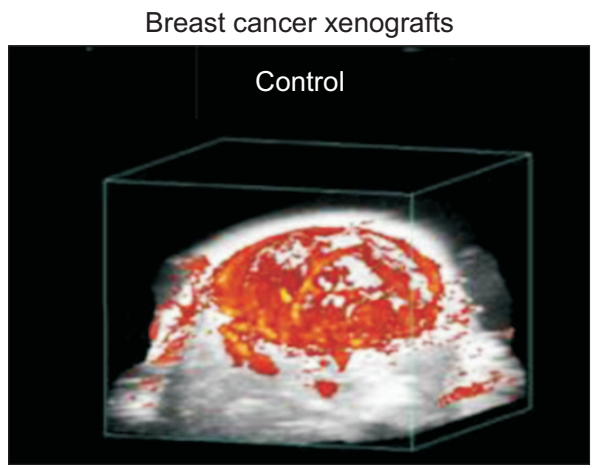

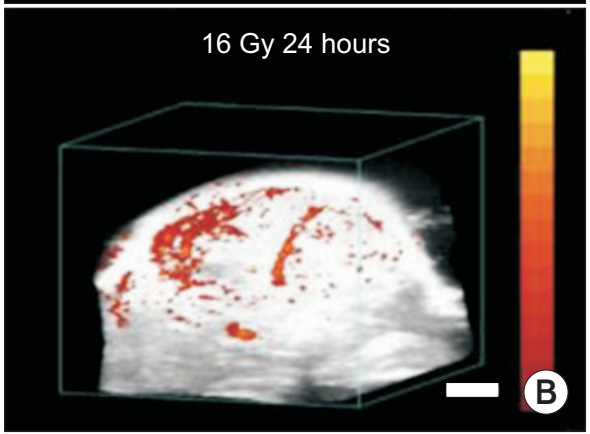

Fig. 4. (A) Angiographs of human melanoma xenografts in nude mice obtained before and 1 week after irradiation with $10 \mathrm{~Gy}$ in a single dose. The necrotic areas are indicated by arrows. Irradiation almost completely abolished the tumor vasculatures. (B) Three-dimensional high-frequency power Doppler ultrasound images of blood flow in MDA-MB-231 human breast cancer xenografts in nude mice. An irradiation with 16 Gy caused drastic reduction in 24 hours.
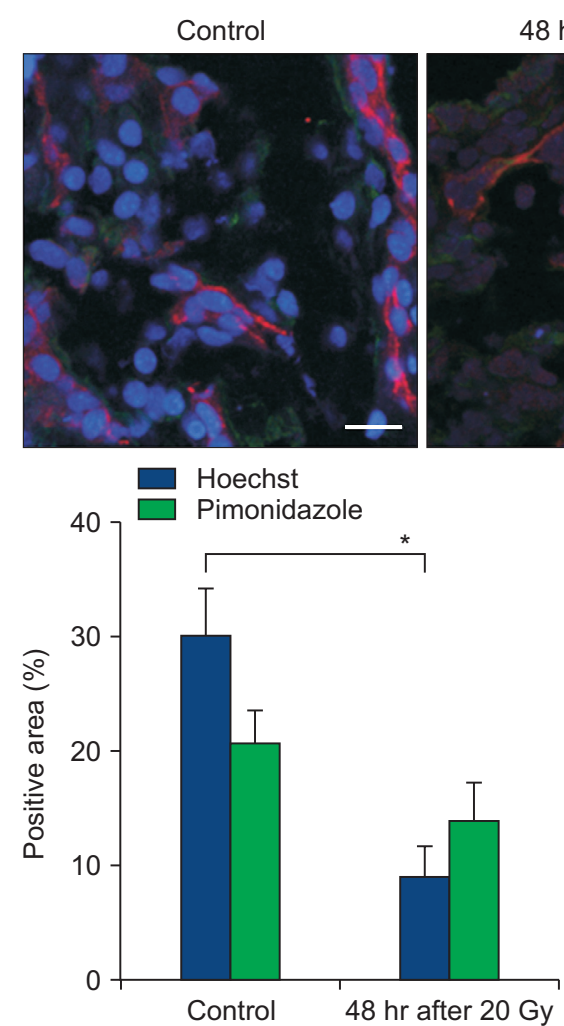

$48 \mathrm{hr}$ after $20 \mathrm{~Gy}$

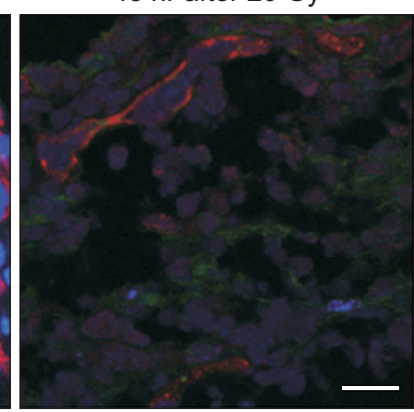

Scale bar, $20 \mu \mathrm{m}$

Red, CD31

Green, pimonidazole Blue, Hoechst

Fig. 5. Immunohistological images of blood perfusion (blue, Hoechst 33342), hypoxia (green, pimonidazole) and endothelial cells (red, CD31 marker) in FSall tumors grown s.c. in the legs of $\mathrm{C} 3 \mathrm{H}$ mice. Tumors were irradiated with $20 \mathrm{~Gy}$ in a single dose and excised 3 days later. Average \pm standard error of 5 tumors are shown. are remarkably radioresistant. For example, the blood vessels in the skin and muscle of rats markedly dilated and blood flow was increased soon after irradiation with 10-60 Gy in a single dose, and remained dilated for longer than 12 days [23]. As mentioned above, the endothelial cells harvested from human breast tumors were radiosensitive as compared to the endothelial cells from the adjacent normal breast tissues. Furthermore, contrary to the defective endothelial walls and chaotic structure of tumor blood vessels, the normal blood vessels consist of uninterrupted lining of endothelial cells supported tightly by basement membranes and pericytes. However it must be noted that endothelial cell damage by high-dose irradiation in normal tissues often result in vascular fibrosis months or years after radiation exposure.

\section{Vascular Damage Causes Indirect Tumor Cell Death}

It has been known for long that vascular damage in tumors caused by high dose irradiation induces tumor cell death. As early as 1932, Cramer [34] reported that vascular damage played an important role in the response of tumors to radiotherapy. Subsequently, Lasnitzki [35] and Merwin et al. [36] observed that irradiation of experimental tumors with 20 to 30 Gy in a single dose induced massive secondary cell death of tumor cells by destroying tumor vasculatures. In 1970s, Clement and his associates $[17,22]$ observed that the 
vascular damages caused by irradiation in Walker tumors of rats or neuroblastoma of mice led to secondary tumor cell death in 2 to 3 days. In order to better understand the potential role of such indirect cell death caused by vascular damage in the response of tumors to SBRT and SRS, Song and his associates $[7,8,10-13]$ recently investigated in detail the kinetics of indirect cell death following high dose irradiation in experimental tumors using the in vivo-in vitro excision assay method. In HT-1080 human fibrosarcoma xenografts in nu/nu mice, the cell survival 3 days after irradiation with $20 \mathrm{~Gy}$ in a single dose was markedly less than that observed immediately after irradiation [11] in agreement with the results in the studies with Walker tumors conducted some 40 years ago [17]. Fig. 6 shows the changes in the cell survival in FSall fibrosarcoma of $\mathrm{C} 3 \mathrm{H}$ mice in 5 days after 10-30 Gy irradiation in a single dose. The cell survival on day 0 is that determined immediately after irradiation and thus the decrease in survival on day 0 represents the cell death caused by the direct effect of radiation. In the tumors irradiated with $10 \mathrm{~Gy}$, the cell survival on days 2, 3, and 5 post-irradiation were similar to that immediately after irradiation. On the other hand, after irradiation with $15 \mathrm{~Gy}$ or $20 \mathrm{~Gy}$, the cell survivals on days 2 and 3 were markedly less than that immediately after irradiation clearly indicating that secondary cell death occurred in 2-3 days after irradiation. On the fifth day after 15-20 Gy irradiation, the cell survivals in some tumors were less than that immediately after irradiation by as much as 2 logs. After irradiation with $30 \mathrm{~Gy}$, the cell survival progressively decreased so that the cell survival in many tumors on day 5 after irradiation was 3-4 logs less than that immediately after irradiation. These recent observations together with the previous reports unequivocally demonstrated that high dose hypo-fractionated irradiation of tumors induces dosedependent indirect cell death most likely by causing vascular destruction thereby deteriorating intratumor environment.

\section{Indirect Tumor Cell Death Due to Immune Response in SBRT and SRS}

It has been reported that high dose hypo-fractionated irradiation promotes anti-tumor immunity [37-40], whereas multi-fractionated radiotherapy with a small dose per fraction
A

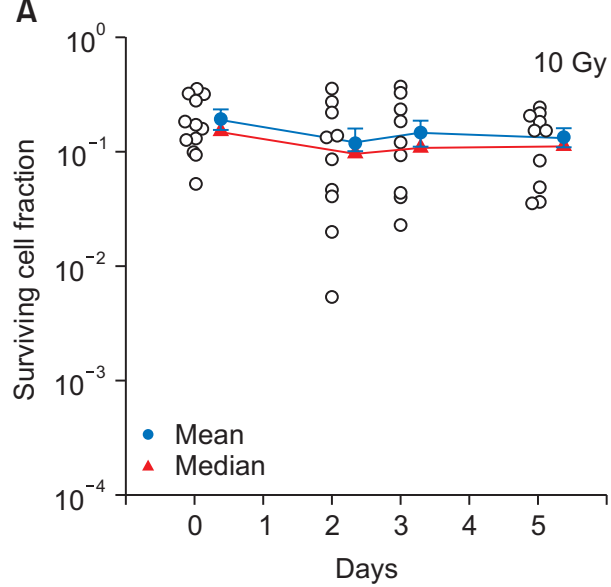

C

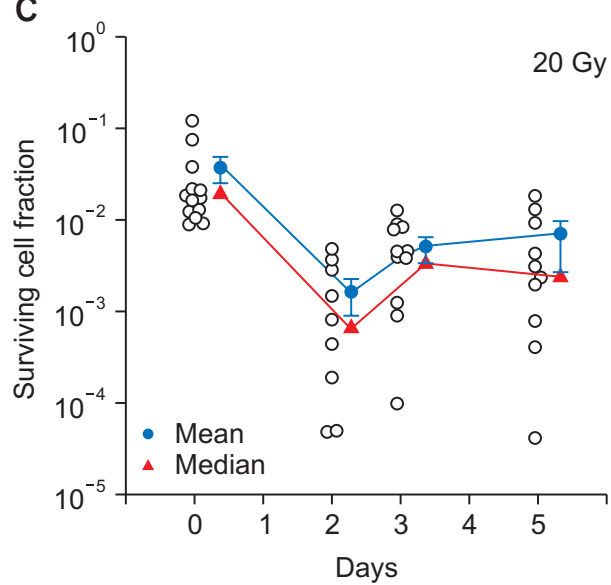

B

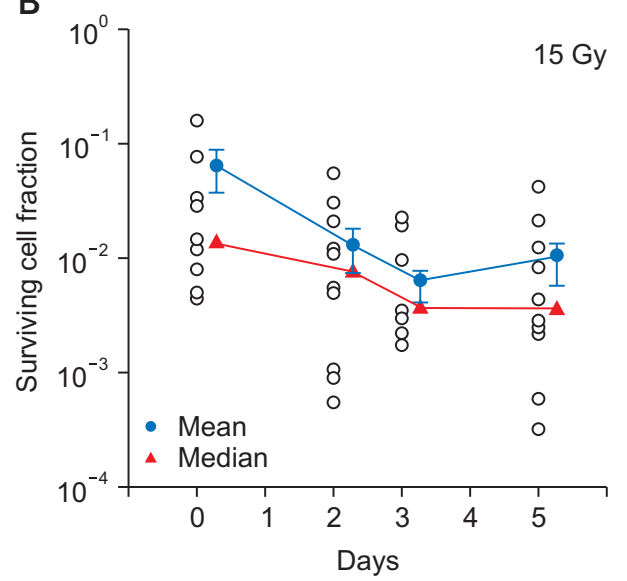

D

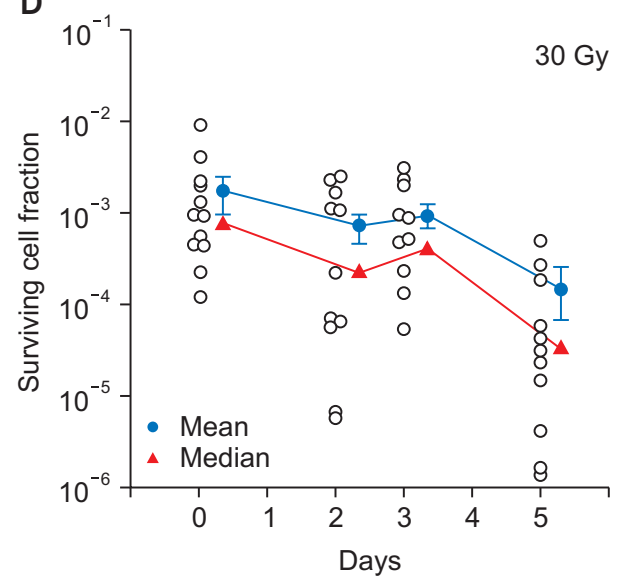

Fig. 6. (A-D) Clonogenic cell survival in FSall tumors grown s.c. in the legs of $\mathrm{C} 3 \mathrm{H}$ mice at $0-5$ days after $10-$ 30 Gy irradiation in a single dose. Cell survival was determined with in vivo-in vitro excision assay method and the surviving cell fractions in irradiated tumors was obtained by normalizing the cell survival in the irradiated tumors to that in the unirradiated control tumors. The open circles indicate the cell survival in each individual tumor. The mean + one standard error (blue) and median (red) are shown. 
suppresses immune competency of the host. Finkelstein et al. [37] attributed the increase in anti-tumor responses by SBRT to elevation of the expression of immune-modulator molecules such as histocompatibility complex, adhesion molecules, heat shock proteins, inflammation mediators, immunomodulating cytokines, and death receptors on the surface of tumor cells. Matsumura et al. [41] reported that irradiation of breast cancers markedly enhanced the secretion of inflammatory chemotactic factor CXCL16, which recruits anti-tumor effector cells including CD8 ${ }^{+}$T-cells. Similarly, irradiated tumor cells induced 'danger' signals by releasing a variety of pro-oxidant and pro-inflammatory cytokines such as tumor necrosis factors (TNFs) and interleukin (IL)-1. The inflammatory cytokines facilitate the uptake of the antigens released from tumor cells by antigen processor cells (APCs), and trigger their maturation and migration to draining lymph nodes. Consistent with these observations, irradiation of B16 melanoma in mice with $15 \mathrm{~Gy}$ in a single dose increased the number of antitumor immune cells by facilitating antigen presentation and priming of antitumor T-cells within draining lymph nodes. Irradiation with $15 \mathrm{~Gy}$ also increased the trafficking of effector T-cells to tumors [42]. Treating tumors with $15 \mathrm{~Gy}$ in a single exposure was more effective than 5 daily irradiations of 3 Gy to increase the above mentioned immune responses. Irradiation of B16 melanoma with 20 Gy dramatically increased T-cell priming in draining lymphoid tissues, and markedly increased the generation of $\mathrm{CD}^{+}$T-cells mediated immunity against the irradiated tumors as well as distant metastases [43]. In a recent phase 1 clinical study [44], metastatic melanoma or renal carcinoma were irradiated with SBRT (20 Gy in 1-3 fractions) and the patients were treated with high-dose IL-2, a cytokine able to increase the generation of immune T-cells. The frequency of proliferating CD4 ${ }^{+}$T-cells and early activated effector memory phenotypes were much higher in the patients treated with SBRT and IL-2 as compared with that in the patients treated with SBRT alone. In the study by Postow et al. [45], an abscopal effect was observed after treating metastatic melanoma with SBRT (9.5 Gy in 3 fractions) in combination with the immune checkpoint inhibitor, ipilimumab.

It is important to point out that the secondary tumor cell death after high dose irradiation as discussed above occurs within 1-3 days after irradiation whereas the full development of radiation-induced tumor-specific immunity usually takes for 1-2 weeks. It is therefore evident that the secondary cell death that occurs soon after tumor irradiation is not caused by an increase in anti-tumor immune response. Furthermore, secondary cell death occurred in 2-3 days after 20 Gy irradiation even in human HT-1080 sarcoma xenografts grown in nu/nu mice despite the fact that the immune system of nul nu mice is considerably compromised [11]. Taken together, it may be concluded that high dose hypo-fractionated irradiation causes ablative cell death directly and indirectly leading to massive release of tumor antigens, thereby elevating antitumor immune response. The anti-immune response, which is elevated 1-2 weeks after tumor irradiation, may not be involved in secondary tumor cells death but it may inhibit the proliferation of the surviving tumor cells leading to suppression of recurrence and metastasis.

\section{Indirect Cell Death Accounts for the High Efficacy of SBRT and SRS}

Fig. 7A shows the radiation survival curves of tumor cells irradiated in vivo demonstrating the contribution of indirect or secondary cell death to the total tumor cell death. The initial steep decline in the cell survival 'a' represents the death of radiosensitive oxic tumor cells. As the radiation dose is further increased, the shape of the survival curve becomes dominated by the death of hypoxic cells characterized by a gradual decline in cell survival. If it is assumed that hypoxic tumor cells are killed only through conventional radiobiological principles, i.e., through direct damage to DNA, the cell survival will decrease linearly with the increase in radiation dose, as indicated by the dotted line 'b'. However, because increasing numbers of tumor cells would be killed as a result of vascular damage with the increase in radiation dose, the radiation survival curve will bend downward as indicated by ' $c$ ' and ' $d$.' This implies that the total cell death by SBRT and SRS with doses higher than about 10 Gy per fraction would be greater than the cell death shown by ' $b$ ', which is assumed to occur when tumor cells are killed only through conventional radiobiological mechanisms. The remarkably high efficacy of SBRT and SRS, which cannot be accounted for by conventional radiobiological estimation may then be attributed to the indirect cell death caused by vascular damage as indicated by ' $c$ ' and 'd' in Fig. 7A.

\section{Reoxygenation, Repair, Repopulation, and Redistribution in SBRT and SRS}

The radiobiological factors which affect the efficacy of fractionated radiotherapy are the so-called 4 Rs (reoxygenation, repair, repopulation, and redistribution). Indications are that the 4 Rs, which are for fractionated irradiation, are not relevant to single dose SBRT or SRS. 
A

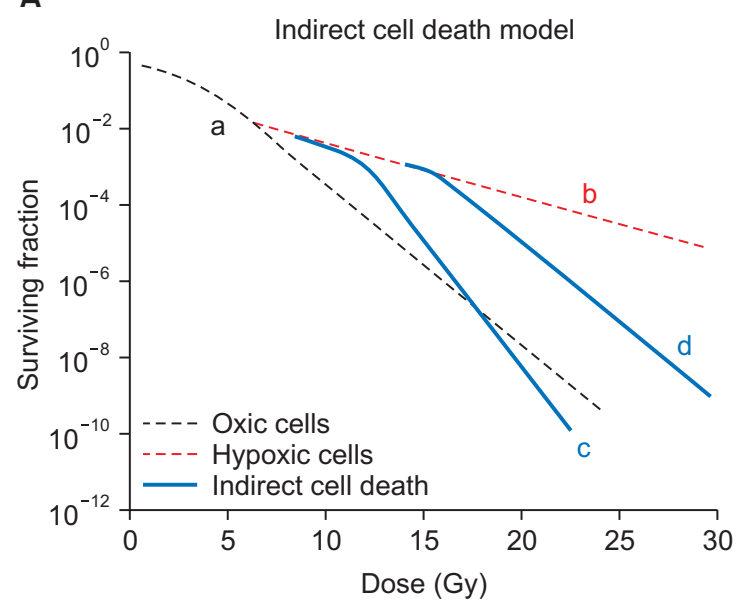

B

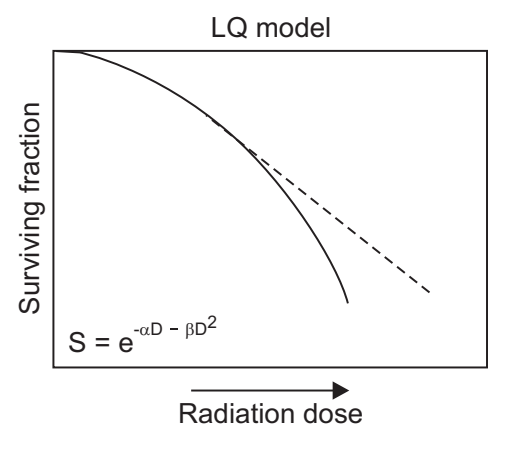

Fig. 7. (A) Hypothetical radiation survival curve of tumor cells in vivo assuming about $10 \%$ of the tumor cells are radiobiologically hypoxic. The 'a' corresponds the radiation-induced death of oxic cells and ' $b$ ' indicates the death of hypoxic cells assuming that radiation-induced cell death is due only to direct damage in DNA/chromosomes. The ' $c$ ' and ' $d$ ' show indirect and additional cell death due to vascular damages at high radiation doses. (B) The dotted line indicates decline in cell survival when radiation-induced cell death is linearly related to radiation dose. Solid line is the linear-quadratic (LO) survival curve which bends downward at high radiation dose indicating that the LO model overestimates cell death at high radiation doses.

\section{Reoxygenation}

Reoxygenation of hypoxic cells in irradiated tumors would occur when blood flow and thus oxygen supply to tumor cells is increased or oxygen consumption is reduced. Given the massive vascular destruction in tumors after high dose irradiation, it is highly unlikely that reoxygenation of hypoxic cells would occur in the tumors within 2-3 days after receiving high dose hypo-fractionated SBRT and SRS. However, it is probable that oxygen consumption would drastically diminish after massive death of tumor cells and thus the surviving hypoxic cells may be reoxygenated. The changes in oxygenation status in tumors following high dose hypofractionated irradiation remains to be elucidated.

\section{Repair}

The half-time for the completion of sub-lethal radiation damage repair in mammalian cells has been reported to be about 30 minutes [46]. Therefore, in treating tumors with SBRT or SRS which take a considerably long irradiation time, repair of sublethal damage may occur during the protracted irradiation. It remains to be investigated how the deterioration of intratumor environment due to vascular damage affects the repair of sublethal radiation damages after high dose irradiation.

\section{Repopulation}

Depletion of cell population by injury, including ionizing radiation, evokes repopulation of cells in both tumors and normal tissues. The time of outset of the compensatory repopulation would vary depending on tissue type and radiation dose. It is known that in fractionated radiotherapy repopulation of tumor cells occurs 2-3 weeks after initiation of radiotherapy. It is conceivable that repopulation of tumor cells may start sooner in ablative SBRT and SRS than that in fractionated radiotherapy.

\section{Redistribution}

In general, irradiation with moderate doses slows down the cell cycle progression through $\mathrm{G} 1$ and $\mathrm{S}$ phases and arrests the cell in $\mathrm{G} 2$ phase in a dose-dependent manner. Fractions of the cells arrested in G2 phase may successfully complete mitosis and progress into $\mathrm{G} 1$ phase or die during mitosis. After irradiation of various cell lines with extremely high doses, i.e., $20 \mathrm{~Gy}$ in a single exposure, cell cycle progression was found to be markedly retarded and many cells died in the cycle phases in which they were irradiated although some of the irradiated cells slowly progressed to G2 phase and died [47]. When HL-60 cells were irradiated with $20 \mathrm{~Gy}$ in a single dose, cells were indefinitely arrested in the cell cycle phases where they were irradiated and died [48].

\section{Linear-Quadratic Model in SBRT and SRS}

The linear-quadratic (LO) model is useful for calculating iso-effect doses in treating cancers with conventional 
multi-fractionated radiotherapy. This model assumes that DNA double-strand break is responsible for the radiationinduced clonogenic cell death and that hypoxic cells are fully reoxygenated during the interval of fractionated irradiation. As shown in Fig. 7B, the $L 0$ survival curve bends downward due to the quadratic component in the formula, and thus the LO model has been suggested to overestimate cell death with the increase in radiation dose. Interestingly, despite the inherent problem with the LO model, some investigators reported that the LQ model fits certain clinical outcomes of SBRT and SRS and thus asserted that direct cell death due to DNA damage alone is sufficient to account for the high clinical efficacy of SBRT and SRS $[9,49]$. As shown in Fig. 7A, the radiation survival curve of tumor cells in vivo also bends downward as the radiation dose is increased above approximately $10 \mathrm{~Gy}$ due to the secondary cell death caused by vascular damage. Therefore, it is conceivable that in certain clinical situation, the calculated cell death by the LO formula may incidentally does not over estimates but approximates the total cell death by SBRT and SRS, which encompasses not only direct and but also indirect cell deaths. Interestingly, the LO model may even underestimate the outcome of SBRT and SRS in situations where indirect cell death is extensive, as is the situation shown by curve ' $d$ ' in Fig. 6 [50]. This implies that the LQ model works for SBRT and SRS in certain clinical situations not because tumor cells are killed only through a direct effect of radiation, but rather because significant fractions of tumor cells are indirectly killed through secondary mechanisms in addition to direct cell death

\section{Conclusion}

Although an increasing number of cancer patients are treated with SBRT and SRS in recent years, the biological mechanisms of these new modalities have been unclear. A simple calculation based on the radiobiological principles for the conventional multi-fractionated radiotherapy clearly suggests that tumor cell death caused by DNA damages by direct effect of radiation alone cannot account for the high efficacy of SBRT and SRS. Evidence now indicates that SBRT and SRS with doses higher than about 10 Gy per fraction induces severe vascular damages in tumors, which then cause secondary and additional tumor cell death. The ensuing degradation of tumor cells would then release massive tumor-specific antigens, thereby elevating anti-tumor immune response leading to suppression of recurrence of tumors and metastasis. The role of 4 Rs and the LQ model is limited in SBRT and SRS. Further understanding of the biological mechanisms of SBRT and SRS would pave a way to potentiate the anti-tumor efficacy of SBRT and SRS.

\section{Conflict of Interest}

No potential conflict of interest relevant to this article was reported.

\section{References}

1. Jang WI, Kim MS, Bae SH, et al. High-dose stereotactic body radiotherapy correlates increased local control and overall survival in patients with inoperable hepatocellular carcinoma. Radiat Oncol 2013:8:250.

2. Nagata Y. Stereotactic body radiotherapy for early stage lung cancer. Cancer Res Treat 2013;45:155-61.

3. Timmerman R, Paulus R, Galvin J, et al. Stereotactic body radiation therapy for inoperable early stage lung cancer. JAMA 2010;303:1070-6.

4. Cho LC, Fonteyne $V$, DeNeve $W$, Lo SS, Timmerman RD. Stereotactic body radiotherapy. In: Levitt SH, Purdy JA, Perez $C A$, Poortmans $\mathrm{P}$, editors. Technical basis of radiation therapy: practical clinical applications. 5th ed. New York, NY: Springer; 2012. p. 363-400.

5. Staehler M, Bader M, Schlenker B, et al. Single fraction radiosurgery for the treatment of renal tumors. J Urol 2015; 193:771-5.

6. Kim YJ, Cho KH, Kim JY, et al. Single-dose versus fractionated stereotactic radiotherapy for brain metastases. Int J Radiat Oncol Biol Phys 2011;81:483-9.

7. Song CW, Park H, Griffin RJ, Levitt SH. Radiobiology of stereotactic radiosurgery and stereotactic body radiation therapy. In: Levitt SH, Purdy JA, Perez CA, Poortmans P, editors. Technical basis of radiation therapy: practical clinical applications. 5th ed. New York, NY: Springer; 2012. p. 51-61.

8. Song CW, Kim MS, Cho LC, Dusenbery K, Sperduto PW. Radiobiological basis of SBRT and SRS. Int J Clin Oncol 2014; 19:570-8.

9. Brown JM, Carlson DJ, Brenner DJ. The tumor radiobiology of SRS and SBRT: are more than the 5 Rs involved? Int J Radiat Oncol Biol Phys 2014;88:254-62.

10. Song CW, Cho LC, Yuan J, Dusenbery KE, Griffin RJ, Levitt $\mathrm{SH}$. Radiobiology of stereotactic body radiation therapy/ stereotactic radiosurgery and the linear-quadratic model. Int J Radiat Oncol Biol Phys 2013;87:18-9.

11. Song CW, Park I, Cho LC, et al. Is indirect cell death involved in response of tumors to stereotactic radiosurgery and stereotactic body radiation therapy? Int J Radiat Oncol Biol Phys 2014;89:924-5. 
12. Sperduto PW, Song CW, Kirkpatrick JP, Glatstein E. A hypothesis: indirect cell death in the radiosurgery era. Int J Radiat Oncol Biol Phys 2015;91:11-3.

13. Song CW, Lee YJ, Griffin RJ, et al. Indirect tumor cell death after high-dose hypofractionated irradiation: implications for stereotactic body radiation therapy and stereotactic radiation surgery. Int J Radiat Oncol Biol Phys 2015;93:166-72.

14. Kocher M, Treuer H, Voges J, Hoevels M, Sturm V, Muller RP. Computer simulation of cytotoxic and vascular effects of radiosurgery in solid and necrotic brain metastases. Radiother Oncol 2000;54:149-56.

15. Park HJ, Griffin RJ, Hui S, Levitt SH, Song CW. Radiationinduced vascular damage in tumors: implications of vascular damage in ablative hypofractionated radiotherapy (SBRT and SRS). Radiat Res 2012;177:311-27.

16. Del Monte U. Does the cell number 10(9) still really fit one gram of tumor tissue? Cell Cycle 2009;8:505-6.

17. Clement JJ, Tanaka N, Song CW. Tumor reoxygenation and postirradiation vascular changes. Radiology 1978;127:799803.

18. Leith JT, Cook S, Chougule $P$, et al. Intrinsic and extrinsic characteristics of human tumors relevant to radiosurgery: comparative cellular radiosensitivity and hypoxic percentages. Acta Neurochir Suppl 1994;62:18-27.

19. Brown JM, Diehn M, Loo BW Jr. Stereotactic ablative radiotherapy should be combined with a hypoxic cell radiosensitizer. Int J Radiat Oncol Biol Phys 2010;78:323-7.

20. Pasqualini R, Arap W, McDonald DM. Probing the structural and molecular diversity of tumor vasculature. Trends Mol Med 2002;8:563-71.

21. Song CW, Levitt SH. Quantitative study of vascularity in Walker carcinoma 256. Cancer Res 1971;31:587-9.

22. Clement JJ, Song CW, Levitt SH. Changes in functional vascularity and cell number following $x$-irradiation of a murine carcinoma. Int J Radiat Oncol Biol Phys 1976;1:671-8.

23. Song CW, Levitt SH. Effect of $x$ irradiation on vascularity of normal tissues and experimental tumor. Radiology 1970;94: 445-7.

24. Song CW, Levitt SH. Vascular changes in Walker 256 carcinoma of rats following $X$ irradiation. Radiology 1971;100:397-407.

25. Wong $\mathrm{HH}$, Song $\mathrm{CW}$, Levitt $\mathrm{SH}$. Early changes in the functional vasculature of Walker carcinoma 256 following irradiation. Radiology 1973;108:429-34.

26. Song CW, Sung JH, Clement JJ, Levitt SH. Vascular changes in neuroblastoma of mice following x-irradiation. Cancer Res 1974;34:2344-50.

27. Emami B, Ten Haken RK, Nussbaum GH, Hughes WL. Effects of single-dose irradiation in tumor blood flow studied by 150 decay after proton activation in situ. Radiology 1981;141:2079.
28. Brown SL, Nagaraja TN, Aryal MP, et al. MRI-tracked tumor vascular changes in the hours after single-fraction irradiation. Radiat Res 2015;183:713-21.

29. Garcia-Barros M, Paris F, Cordon-Cardo C, et al. Tumor response to radiotherapy regulated by endothelial cell apoptosis. Science 2003;300:1155-9.

30. Solesvik OV, Rofstad EK, Brustad T. Vascular changes in a human malignant melanoma xenograft following single-dose irradiation. Radiat Res 1984;98:115-28.

31. El Kaffas A, Giles A, Czarnota GJ. Dose-dependent response of tumor vasculature to radiation therapy in combination with Sunitinib depicted by three-dimensional high-frequency power Doppler ultrasound. Angiogenesis 2013;16:443-54.

32. Kioi M, Vogel H, Schultz G, Hoffman RM, Harsh GR, Brown JM. Inhibition of vasculogenesis, but not angiogenesis, prevents the recurrence of glioblastoma after irradiation in mice. J Clin Invest 2010;120:694-705.

33. Chen FH, Chiang CS, Wang CC, et al. Radiotherapy decreases vascular density and causes hypoxia with macrophage aggregation in TRAMP-C1 prostate tumors. Clin Cancer Res 2009;15:1721-9.

34. Cramer W. Experimental observations on the therapeutic action of radium. In: The 10th Scientific Report on Imperial Cancer Research Fund. London: The Fund; 1932. p. 95-123.

35. Lasnitzki I. A quantitative analysis of the direct and indirect action of $X$ radiation on malignant cells. $\mathrm{Br} J$ Radiol 1947;20: 240-7.

36. Merwin R, Algire GH, Kaplan HS. Transparent-chamber observations of the response of a transplantable mouse mammary tumor to local roentgen irradiation. J Natl Cancer Inst 1950;11:593-627.

37. Finkelstein SE, Timmerman R, McBride WH, et al. The confluence of stereotactic ablative radiotherapy and tumor immunology. Clin Dev Immunol 2011;2011:439752.

38. McBride WH, Schaue D. In situ tumor ablation with radiation therapy: its effect on the tumor microenvironment and antitumor immunity. In: Keisari Y, editor. Tumor ablation: effects on systemic and local anti-tumor immunity and on other tumormicroenvironment interactions. New York, NY: Springer; 2013. p. 109-19.

39. Kaur P, Asea A. Radiation-induced effects and the immune system in cancer. Front Oncol 2012;2:191.

40. Ahmed MM, Guha C, Hodge JW, Jaffee E. Immunobiology of radiotherapy: new paradigms. Radiat Res 2014;182:123-5.

41. Matsumura S, Wang B, Kawashima N, et al. Radiation-induced CXCL16 release by breast cancer cells attracts effector T cells. J Immunol 2008;181:3099-107.

42. Lugade AA, Moran JP, Gerber SA, Rose RC, Frelinger JG, Lord EM. Local radiation therapy of B16 melanoma tumors increases the generation of tumor antigen-specific effector cells that traffic to the tumor. J Immunol 2005;174:7516-23. 
43. Lee $Y$, Auh SL, Wang $Y$, et al. Therapeutic effects of ablative radiation on local tumor require $\mathrm{CD} 8+\mathrm{T}$ cells: changing strategies for cancer treatment. Blood 2009;114:589-95.

44. Seung SK, Curti BD, Crittenden $M$, et al. Phase 1 study of stereotactic body radiotherapy and interleukin-2: tumor and immunological responses. Sci Transl Med 2012;4:137ra74.

45. Postow MA, Callahan MK, Barker CA, et al. Immunologic correlates of the abscopal effect in a patient with melanoma. $\mathrm{N}$ Engl J Med 2012;366:925-31.

46. Fowler JF, Welsh JS, Howard SP. Loss of biological effect in prolonged fraction delivery. Int J Radiat Oncol Biol Phys 2004; 59:242-9.

47. Jeong JH, Park IW, Kang MA, Kim MS, Song CW. Effect of high- dose hypofractionated irradiation (SBRT/SRS) on cell cycle progression [abstract]. In: 61th Radiation Research Society Annual Meeting; 2015 Sep 19-22; Weston, FL. Abstract no. PS1-24.

48. Park H, Lyons JC, Griffin RJ, Lim BU, Song CW. Apoptosis and cell cycle progression in an acidic environment after irradiation. Radiat Res 2000;153:295-304.

49. Brenner DJ. The linear-quadratic model is an appropriate methodology for determining isoeffective doses at large doses per fraction. Semin Radiat Oncol 2008;18:234-9.

50. Kirkpatrick JP, Meyer JJ, Marks LB. The linear-quadratic model is inappropriate to model high dose per fraction effects in radiosurgery. Semin Radiat Oncol 2008;18:240-3. 